\title{
NGC 2587: a sparse open cluster projected onto a populous star field
}

\author{
Andrés E. Piatti, ${ }^{1}$ Juan J. Clariá ${ }^{2}$ and Andrea V. Ahumada ${ }^{2,3}$ \\ ${ }^{1}$ Instituto de Astronomía y Física del Espacio, Casilla 67, Suc. 28, 1428 Buenos Aires, \\ Argentina \\ email: andres@iafe.uba.ar \\ ${ }^{2}$ Observatorio Astronómico de Córdoba, Laprida 854, 5000 Córdoba, Argentina \\ email: claria@oac.uncor.edu \\ ${ }^{3}$ European Southern Observatory, Alonso de Córdova 3107, Santiago, Chile \\ email: aahumada@eso.org
}

\begin{abstract}
We present CCD $B V I_{\mathrm{KC}}$ photometry in the field of the open cluster NGC 2587. We developed a new method to clean statistically the colour-magnitude diagrams. NGC 2587 is found to be slightly younger than the Hyades and probably of solar metallicity. From 18 probable members with measured proper motions, we derived the following mean cluster values:

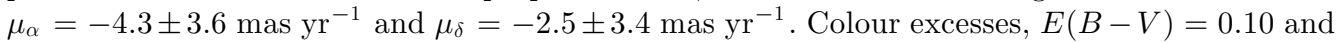
$E(V-I)=0.15 \mathrm{mag}$, and a heliocentric distance of $3.7 \pm 0.7 \mathrm{kpc}$ are obtained. The interstellar extinction in the cluster direction is found to follow the normal reddening law.
\end{abstract}

Keywords. open clusters and associations: individual (NGC 2587), techniques: photometric

\section{Introduction}

No photometric study has yet been published of NGC 2587, a relatively bright open cluster in Puppis. This is one of the reasons that justifies the current work. Although Bustos Fierro (2007) (hereafter BF07) measured proper motions within a $2^{\circ} \times 2^{\circ}$ field of a Carte du Ciel plate containing NGC 2587, he neither determined the basic cluster parameters nor verified its physical reality. The present study attempts at shedding light on the nature of this object by using high-quality photometric data obtained with the $0.9 \mathrm{~m}$ telescope at Cerro Tololo Inter-American Observatory (Chile).

\section{Cluster centre and angular radius}

The coordinates of the cluster centre were determined by fitting Gaussian distributions to the stellar-density profiles in the $X$ and $Y$ directions, using tasks in IRAF and applying the statistical method described by Piatti et al. (2006). The coordinates of the cluster centre were $\left(X_{\mathrm{c}}, Y_{\mathrm{c}}\right)=(1300 \pm 10,1190 \pm 10)$ pixels, equivalent to $\alpha(2000)=$ $8^{\mathrm{h}} 23^{\mathrm{m}} 27^{\mathrm{s}}, \delta(2000)=-29^{\circ} 30^{\prime} 34^{\prime \prime}$. Although these values agree well with those estimated by eye, BF07 and Palma et al (2008) centred the cluster $5.9^{\prime}$ to the southeast and $1.2^{\prime}$ to the northeast from our value, respectively. The radial stellar-density profile of NGC 2587 is not very well approximated by a Gaussian. However, $75 \%$ of the cluster members (see Section 3) are found to be located within $5^{\prime}$ from the cluster centre. Since the most distant member lies at about $8^{\prime}$ from this centre, we adopted the latter value as a rough cluster radius estimate. 


\section{A new statistical method to clean colour-magnitude diagrams}

Figure 1 shows the colour-magnitude diagrams (CMDs) of all observed stars in the cluster field. It is difficult to accurately identify the cluster main sequence (MS) from the CMDs: a crowded MS rises up from the magnitude limit at $V \sim 16$. A few apparent clumps of red stars are also identified at $(V, V-I) \sim(12.5,1.2),(13.2,1.5)$ mag as well as their counterparts in the $(V, B-V)$ CMD. From the various MSs possible, that of the cluster appears to be the one which reaches the brightest magnitude and bluest colour. Thus, if we want to estimate the cluster's fundamental parameters from the CMDs, we must first disentangle the cluster MS stars from those belonging to the surrounding field. Note that both cluster and field stars are affected by nearly the same reddening, which precisely causes the overlap of their MSs. This becomes an additional difficulty when attempting to clean the cluster CMDs. NGC 2587 does not appear to be a concentrated or rich stellar aggregate. On the contrary, bearing in mind the cluster appearance on the sky and the results obtained by BF07 and Palma et al. (2008), the apparently small number of cluster stars would be spread over the field. Precisely due to the low density and sparse distribution of cluster stars and to the populous star field onto which the cluster is projected, any cluster CMD constructed from circular extractions around its centre would be highly contaminated by field stars. Since NGC 2587 seems to cover most of the observed $13.6^{\prime} \times 13.6^{\prime}$ area, we developed an alternative method to clean statistically the CMDs, separately. The method relies on the following assumptions: (i) the cluster is a low-stellar-density object, (ii) cluster stars are spread throughout the entire observed field and (iii) the cluster is projected onto a populous star field. Considering these three assumptions on the whole implies that any extracted CMD of stars located within a unit area at any place of the observed field should be mainly represented by the stellar field CMD. Thus, we divided the observed region covering [0:2000,0:2000] pixels into 64 boxes of 250 pixels on a side and constructed for each the corresponding CMDs. At first glance, there are some differences among the various box-extracted CMDs, which reveals a certain inhomogeneity in the spatial distribution of the stellar brightness and colours.

The proposed method consists of adopting one of the 64 box-extracted CMDs as a 'starfield CMD' to statistically clean the remaining 63 box-extracted CMDs. We repeated the cleaning process using as star-field CMD each of the 64 CMDs. At the end of this process, each box-extracted CMD was individually cleaned using a different star-field CMD each
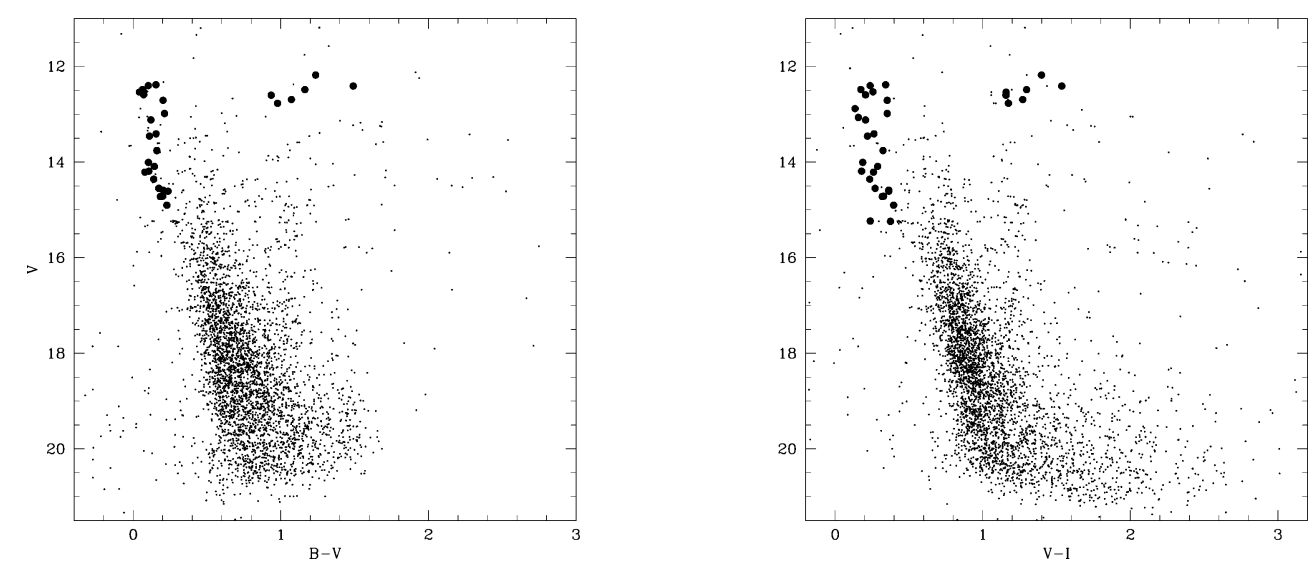

Figure 1. $(V, B-V)$ and $(V, V-I)$ diagrams for the stars measured in the field of NGC 2587 . Filled circles represent stars adopted as probable cluster members. 
time. The cleaning was performed using bins of $[\Delta V, \Delta(B-V)]=[\Delta V, \Delta(V-I)]=$ $(1.0,0.2)$ mag. We carried out the cleaning using bins of $(0.5,0.2) \mathrm{mag}$ and $(0.5,0.1)$ mag, as well as boxes of $500 \times 500$ pixels to compare residuals. When comparing the 63 cleaned CMDs for a given box with the observations, we find the residuals from boxto-box variations and the fiducial CMD features of that box. In other words, a star that appears nearly 63 times in the cleaned CMDs of a given box has many chances (statistically speaking) of being part of the intrinsic characteristics of the CMD of that box. Moreover, a star that is eliminated nearly 63 times has magnitude and colours within the typical values found in the field-star CMDs. Thus, the fewer times a star is removed in a given box, the higher its probability of being an inherent characteristic of that box. Therefore, we decided to adopt as a probable cluster member of NGC 2587, any star that has at least 2 measures of $(B-V)$ and $(V-I)$, and has also been removed fewer than $20 \%$ of the times. From the 4406 measured stars, a total of 32 fulfill such requirements. Figure 2 shows the resulting cleaned CMDs (filled circles) superimposed onto the observed data (dots). Among the observed stars brighter than $V=12$ mag, only HD 70927 has a known MK spectral type. According to Houk \& Cowley (1975), this is an F7/8 II-type star. Therefore, its visual absolute magnitude is $M_{V}=-2.3$ (Straizys 1992). Assuming HD 70927 were affected by the same reddening as NGC 2587, it would clearly be a foreground object located $\sim 1.4 \mathrm{kpc}$ from the Sun. As for the remaining observed bright stars, their positions in the CMDs indicate that they are very unlikely to be members.

\section{Cluster fundamental parameters}

We fitted theoretical isochrones of Lejeune \& Schaerer (2001) to the cleaned CMDs. First, we independently fitted the zero-age main sequence (ZAMS) to the $(V, B-V)$ and $(V, V-I)$ CMDs for each selected metallicity and derived $E(B-V), E(V-I)$ and $V-M_{V}$. The fit for $Z=0.020$ best matches the nonevolved stellar sequence. Next, we selected isochrones of $\log t(\mathrm{yr})$ greater than 8.0 and used the derived pairs of $\left[V-M_{V}, E(B-V)\right]$ and $\left[V-M_{V}, E(V-I)\right]$ values to estimate the cluster's age. The isochrone of $\log t(\mathrm{yr})=$ $8.70(t=500 \mathrm{Myr})$ turned out to most accurately reproduce the cluster features. To match
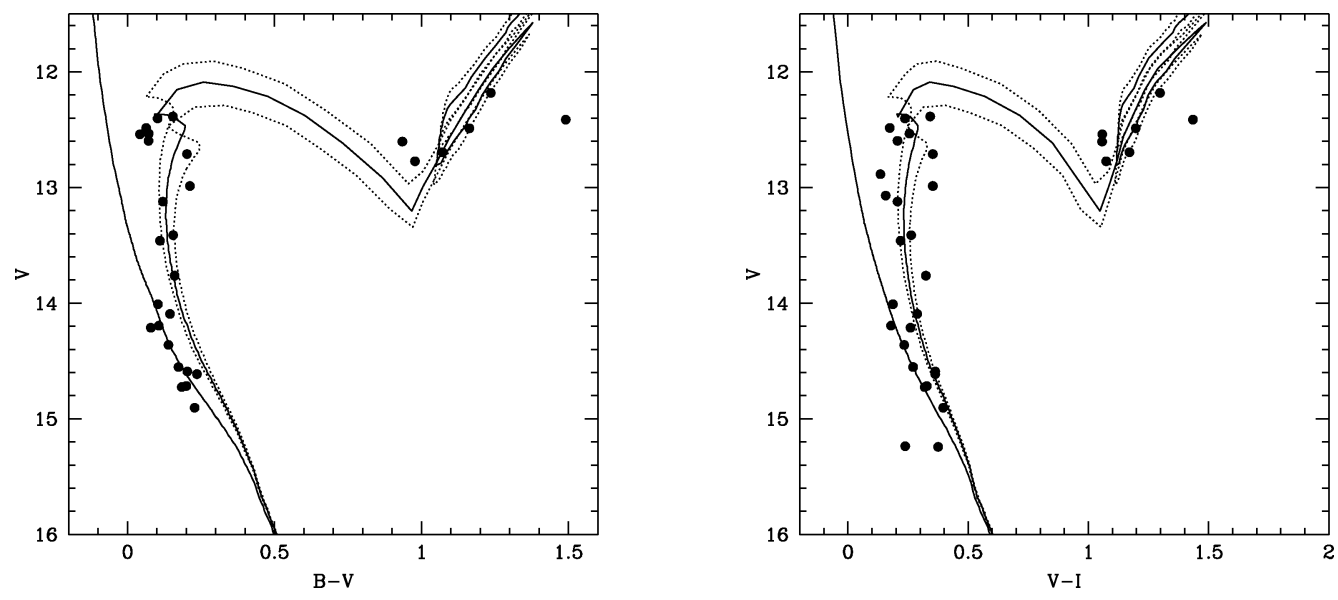

Figure 2. $(V, B-V)$ and $(V, V-I)$ diagrams for the probable members of NGC 2587. The zero-age main sequence and the adopted isochrones from Lejeune \& Schaerer (2001), computed taking into account overshooting, are overplotted as solid lines. For comparison purposes, we included as dotted lines the isochrones associated with the cluster's age uncertainties. 
this isochrone, we used $E(B-V), E(V-I)$ and $V-M_{V}$ values of $0.10,0.15$ and $12.50 \mathrm{mag}$, respectively, which were derived from the ZAMS fit. In Figure 2 we overplot the ZAMS and the isochrone of $\log t(\mathrm{yr})=8.70$ (solid lines) for $Z=0.020$ on the cluster CMDs and two additional isochrones of $\log t(\mathrm{yr})=8.65$ and 8.75 (dotted lines), for comparison purposes. We compared the observed $E(V-I) / E(B-V)$ ratio $(=1.50 \pm 0.40)$ with that from the normal extinction law (Dean et al. 1978) and found very good agreement. We used $R=A_{V} / E(B-V)=3.2$ (Straizys 1992) to derive a cluster heliocentric distance of $2.7 \pm 0.7 \mathrm{kpc}$ and a distance from the Galactic plane of $210 \mathrm{pc}$. Assuming the Sun's distance from the Galactic centre to be $8.5 \mathrm{kpc}$, we derived a Galactocentric distance to the cluster of $\sim 9.8 \mathrm{kpc}$.

\section{Analysis of proper motions}

BF07 measured proper motions (PMs) of 4172 stars in the cluster region. We found that 20 of our 32 probable members are in his list. Except for two stars with remarkably different $\mu_{\delta}$ values, the remaining 18 stars have rather similar PMs, with average values of $\mu_{\alpha}=-4.3 \pm 3.6$ and $\mu_{\delta}=-2.5 \pm 3.4$ mas $\mathrm{yr}^{-1}$. This result is in excellent agreement with the membership status adopted for these stars. For this reason, we assumed these mean values as the cluster mean PMs. The bright star HD 70927, considered to be a nonmember owing to its $M_{V}$, has a motion which differs by a little more than $1 \sigma$ from the motion accepted for the cluster members. BF07 derived membership probabilities between 50 and $70 \%$ for 23 stars which he considered cluster members. However, only two of these stars appear to be located along the isochrone which best reproduces the cluster features. The remaining 21 stars apparently belong to the foreground/background field. Since BF07 constructed the cluster PM distributions from stars spread across a region which includes part of the cluster area and part of the field region, the resulting mean PMs do not represent those of the cluster, but are a mixture of cluster and field PMs.

\section{References}

Bustos Fierro, I. H. 2007, PhD Thesis, Universidad Nacional de Córdoba, Argentina (BF07) Dean, F. J., Warren, P. R., \& Cousins, A. W. J. 1978, MNRAS, 183, 569

Houk, N. \& Cowley, A. P. 1975, University of Michigan Catalogue of Two-Dimensional Spectral Types for the HD stars

Lejeune, T. \& Schaerer, D. 2001, A\& A, 366, 538

Palma, T., Ahumada, A. V., Clariá, J. J., \& Bica, E. 2008 AN, 329, 392

Piatti, A. E., Clariá, J. J., \& Ahumada, A. V. 2006 MNRAS, 367, 599

Straizys, V. 1992 Multicolor Stellar Photometry, Pachart Publishing House: Tucson, Arizona 